\title{
Treatment of the cardiac hypertrophic response and heart failure with ginseng, ginsenosides and ginseng-related products
}

\begin{tabular}{|r|l|}
\hline Journal: & Canadian Journal of Physiology and Pharmacology \\
\hline Manuscript ID & cjpp-2017-0092 \\
\hline Manuscript Type: & Review \\
\hline Date Submitted by the Author: & $22-$ Feb-2017 \\
\hline Complete List of Authors: & $\begin{array}{l}\text { Karmazyn, Morris; 1118 Lawson Road } \\
\text { Gan, Tracey; 1118 Lawson Road }\end{array}$ \\
\hline Keyword: & ginseng, ginsenosides, cardiac hypertrophy, heart failure \\
\hline \multicolumn{2}{|l}{} \\
\hline
\end{tabular}

\section{SCHOLARONE ${ }^{m}$}

Manuscripts 
Treatment of the cardiac hypertrophic response and heart failure with ginseng, ginsenosides and ginseng-related products

Morris Karmazyn and Xiaohong Tracey Gan

London, Ontario, Canada N6G 2X6 


\begin{abstract}
Heart failure is a major medical as well as economic burden throughout the world. Although various treatment options are available to treat heart failure, death rates in both men and women remain high. Potential adjunctive therapies may lie with use of herbal medications many of which possess potent pharmacological properties. Among the most widely studied is ginseng, a member of the genus Panax which is grown in many parts of the world and which has been used as a medical treatment for a variety of conditions for thousands of years, particularly in Asian societies. There are a number of ginseng species each possessing distinct pharmacological effects due primarily to differences in their bioactive components including saponin ginsenosides and polysaccharides. While experimental evidence for salutary effects of ginseng on heart failure is robust clinical evidence is less so primary due to a paucity of large scale well controlled clinical trials. However, there is evidence from small trials that ginseng-containing Chinese medications such as Shenmai can offer benefit when administered as adjunctive therapy to heart failure patients. Substantial additional studies are required, particularly in the clinical arena, to provide evidence for a favourable effect of ginseng in heart failure patients.
\end{abstract}

Key words: ginseng, ginsenosides, cardiac hypertrophy, heart failure 


\section{Introduction}

Heart failure represents a major medical burden on an international scale with approximately twenty six million individuals afflicted with this condition worldwide (Ambrosy et al. 2014). Heart failure is the final common pathway for numerous cellular and molecular defects caused by many instigating factors including myocardial infarction, genetic factors, diabetes, pulmonary hypertension, as well as others. Prognosis is generally poor with a 50\% mortality rate within five years from initial diagnosis. Although hospital admission rates are generally equally divided between males and females, mortality rates for women are markedly higher than those observed for men (Tu et al. 2009). An important component of the heart failure process is ventricular remodelling as exemplified by changes in ventricular geometric architecture as a response to insult which eventually results in, or at least contributes to, defective ventricular function. As such, myocardial remodelling represents an important therapeutic target to mitigate deterioration of ventricular function after insult. Although remodelling can occur early after insult, it is generally considered to be a chronic event which progressively increases in severity (Frantz et al. 2009; Opie et al. 2006; Sun 2009). Remodelling is associated with various aspects of pathology such as hypertrophy, extracellular matrix deposition, as well as what may be considered as cellular remodelling which encompasses defective cell function including ionic regulation, generation of reactive oxygen species, energy production and substrate utilization (Frantz et al. 2009; Opie et al. 2006; Sun 2009). Cellular remodelling, especially as this pertains to changes in mitochondrial dynamics, represents an important component of the remodelling program and one which may determine reversibility of remodelling and functional recovery following infarction (Javadov and Karmazyn 2007). Early hypertrophic responses are one of the major compensatory 
mechanisms to myocardial injury (Katz 2000). However, prolonged cardiac hypertrophy is, paradoxically, one of the major contributors to the myocardial remodelling processes leading up to heart failure.

Extensive research is ongoing which attempts to define the molecular mechanisms that contribute to or underlie cardiac hypertrophy and remodelling (reviewed by Shimizu and Minamino 2016). Although the process of cardiac hypertrophy is extremely complex one very central mechanism involves the activation of calcineurin, a calcium stimulated protein phosphatase which can dephosphorylate a number of targets. Among the most important of these targets is the transcriptional factor nuclear factor of activated T cells or NFAT, which when dephosphorylated is able to translocate into the nucleus to initiate transcription and the hypertrophic process (Heineke and Molkentin 2006). Thus, calcineurin inhibition represents a potentially effective therapeutic target for reducing myocardial hypertrophy, remodelling and heart failure (Panther et al. 2009). Indeed, prevention of hypertrophy, and as has become evident recently, its reversal, represent important therapeutic goals for the treatment of heart failure (Frey et al. 2004). As such, identifying novel approaches to reduce or even reverse the hypertrophicremodelling process represents important goals for the development of effective therapeutic strategies for treating heart failure. There is substantial evidence, primarily from experimental studies, that ginseng could be a suitable candidate in this regard although clinical evidence is not robust, as discussed below.

\section{Experimental models to study heart failure}

Various experimental models can be employed, using both in vitro and in vivo approaches, to determine antihypertrophic and remodelling effects of potential novel therapeutic agents. 
Among these include the use of cultured cardiomyocytes exposed to pro-hypertrophic stimuli of clinical significance such as angiotensin II, endothelin-1 or adrenergic agents acting on either myocardial $\beta_{1}$ or $\alpha_{1}$ receptors. In addition, various animal models are good surrogates for clinical heart failure including surgical models of heart failure such as those produced by sustained infarction subsequent to coronary artery occlusion or pressure overload-induced heart failure produced by aortic banding. Moreover, there are genetic models of heart failure which could provide useful information particularly related to gene-specific regulation of the hypertrophic and remodelling programs which contributes to heart failure. These models including a discussion of their appropriateness for the study of clinical heart failure have been the subject of various published reviews (Breckenridge 2010; Muders and Elsner 2000; Patten and Hall-Porter 2009).

\section{Ginseng: a brief introduction}

Ginseng is a widely used herbal product which has been employed as a medication in Asian societies for thousands of years and whose popularity and use are increasing in western cultures. Ginseng is not a single product and indeed it exhibits extreme complexity by virtue of the fact that it is represented by several species and, as discussed below, contains a myriad of bioactive active compounds the nature of which generally reflects the type of ginseng species. Moreover, many of the bioactive saponin components of ginseng undergo biotransformation to novel compounds after ingestion, mostly by the actions of gut bacterial enzymes resulting the production of novel compounds with potent biologic effects. As such, many of the effects of ginseng in vivo may not be due to its original constituents but rather to its metabolic products. 
Ginseng belongs to the Araliaceae family and has several different species such as Panax ginseng (Asian ginseng), Panax japonicas (Japanese ginseng), and Panax quinquefolius (North American ginseng) among others. Panax ginseng is also referred to as $P$ ginseng C.A. Meyer named after Carl Anton von Meyer, the German-born Russian botanist who identified the different ginseng species in 1842. This species of ginseng can be found naturally in forests of large shady trees in dense and nutrient-limited soil in China and Korea. P quinquefolius is grown under relatively similar conditions in North America, particularly southern Canada including the provinces of Ontario, especially the southwestern part of the province and Québec as well as in the United States, principally in the state of Wisconsin.

As mentioned above the complexity of ginseng is reflected by the fact that it can contain up to 200 active ingredients depending on the ginseng type with Korean red ginseng (basically $P$ ginseng which has been subjected to a heating protocol thus altering its component profile thereby possibly improving its biological potency) possessing the most constituents, among these being polysaccharides, polyacetylenes, peptides and amino acids (Attele et al. 1999). However, the major active components in ginseng are the saponin compounds which were first identified in 1854 (Garriques 1854) although their structures were elucidated much more recently (Shibata et al.1963a, 1965a, 1965b). At present, dozens of saponin ginsenosides have been identified in various components of the ginseng plant including root, leaves, flower buds and seeds although key ginsenosides exerting biological effects including ginsenosides $\mathrm{Rb} 1, \mathrm{Rb} 2, \mathrm{Rc}, \mathrm{Rd}, \mathrm{Re}, \mathrm{Rf}$, and Rg1 are most abundant in the ginseng root (Shin et al. 2015). Indeed, ginsenoside nomenclature is based on a rather simple formula, $\mathrm{Rx}$, where " $\mathrm{R}$ " represents "root" and " $\mathrm{x}$ " indicates the polarity of the ginsenoside based on HPLC analyses in alphabetic order (Shibata et al. 1963b). For example, $\mathrm{Rg}$ is more polar (hydrophilic) than $\mathrm{Ra}$ which is the least polar and 
therefore more hydrophobic of all the ginsenosides. Table 1 compares the component profile of various ginseng species.

As already alluded to it is important to reinforce the concept of biotransformation of ginseng constituents following oral ingestion of ginseng. Indeed, the main bioactive constituents in ginseng including both saponins and polysaccharides are not easily absorbed from the intestine when ingested due to the hydrophilic nature of these compounds as well as their molecular size and therefore they can undergo metabolism and biotransformation by bacteria in the gastrointestinal tract (Kim 2002; Kobashi and Akao 1997). In fact, less than five percent of major ginsenosides can be identified in serum following ingestion of ginseng in the rat (Odani et al. $1983 a, 1983 b)$. Among the most studied of these biotransformation reactions is the synthesis of the biologically active 20-O-( $\beta$-D-glucopyranosyl)-20(S)-protopanaxadiol generally referred to as compound $\mathrm{K}$ which can be produced from various ginsenosides including $\mathrm{Rb} 1, \mathrm{Rb} 2$ and $\mathrm{Rc}$ by intestinal bacteria (Akao et al. 1998; Karikura et al. 1990, 1991).

\section{Ginseng for treating heart failure}

One of the first English-language reports suggesting that ginseng may offer benefit in heart failure was a study published in 1992 demonstrating that a compound called Kyushin improves cardiac function in a rabbit model of acute heart failure produced by protease application to the surface of the left ventricular anterior wall (Morishita et al. 1992a). Kyushin however contains a number of natural products with potential therapeutic properties including toad venom although the primary constituent is ginseng. However, the precise contribution of ginseng, relative to the other constituents, to the improved function cannot in this heart failure model is not completely known. In addition, Kyushin produces a number of direct cardiovascular effects including 
cardiostimulatory and arrhythmogenic effects (Morishita et al. 1992b). Interestingly, Kyushin is currently available commercially and marketed as a "heart tonic" for a variety of conditions.

$P$ ginseng has been shown to afford various beneficial effects against heart failure produced by the antineoplastic agent adriamycin when administered for two weeks to rats. Among the salutary effects were found to include reduced mortality, improved cardiac function and improved myocardial antioxidant status (You et al. 2005). Indeed, among the major limitations for the use of adriamycin as a cancer treatment is the drug's substantial cardiotoxicity. Thus it would be of importance and clinical relevance to assess whether the beneficial effect of ginseng is associated with any effect on adriamycin's antineoplastic properties.

In terms of individual ginsenosides, these as well have been shown to reduce heart failure in a number of experimental models involving both left and right ventricular hypertrophy and failure. The aortic banding or coarctation model of heart failure is a widely used preparation to study cardiac hypertrophy and heart failure caused by pressure overload and one which has been used with different animal species, although with respect to ginseng-related studies virtually all of these investigations were performed using rat models. Generally speaking, animals subjected to aortic banding develop relatively rapid left ventricular hypertrophy and heart failure which is dependent on the degree or severity of aortic banding. Ginsenoside Rgl appears to be the only ginsenoside studied in this model of heart failure although as discussed below ginsenoside Rb1 has also been shown to exert benefit in other heart failure models. Ginsenoside Rg1 has been shown to produce a marked reduction in the hypertrophic response in rats subjected to 21 days of abdominal aorta banding, an effect which was associated with suppression in the upregulation in pro-hypertrophic cell signalling mediated including ERK mitogen activated protein kinase (MAPK) and calcineurin (Deng et al. 2009). In a separate study by the same group and using a 
similar model and treatment protocol the potential role for nitric oxide (NO) was also proposed since the beneficial effect of Rg1 was abolished by the NO synthase inhibitor L-NAME and downregulation of endothelial NO synthase expression by aortic banding was prevented by Rg1 (Deng et al. 2010). Rg1 also was found to reduce myocardial fibrosis and enhance angiogenesis in rats subjected to four weeks of thoracic aortic banding which was associated with improved left ventricular function (Zhang et al. 2013). It was proposed that the salutary effect of $\operatorname{Rg} 1$, at least with respect to increased angiogenesis was related to the upregulation in expression of vascular endothelial growth factor (VEGF) as well as hypoxia-inducible factor 1 (HIF-1), a transcriptional factor implicated in the angiogenesis response.

The sustained coronary artery occlusion model is also an extensively used approach to produce heart failure secondary to myocardial infarction. Using this approach, the authors' laboratory has demonstrated that oral administration of $P$ quinquefolius can effectively inhibit the progression of heart failure in rats and diminish hypertrophy in the surviving left ventricle (Guo et al. 2011). This effect was attributed to the inhibition of the isoform 1 sodium-hydrogen exchanger (NHE-1) an electroneutral membrane-bound intracellular $\mathrm{pH}$ regulatory process which extrudes one proton in exchange for sodium influx (Karmazyn et al. 2008). The influx of sodium would then increase intracellular calcium levels through the sodium-calcium exchanger, either by slowing the removal of calcium or by inducing a reverse-mode sodium-calcium exchange activity, which in turn would activate calcineurin activity resulting in the hypertrophic response (Karmazyn et al. 2008). Indeed, hearts from animals subjected to coronary artery ligation treated with $P$ quinquefolius demonstrated a marked blunting of NHE-1 expression and activity, calcineurin activation and translocation of the transcription factor NFAT into nuclei (Guo et al. 2011). Thus, $P$ quinquefolius appears to be an affective NHE-1 inhibitor. It is 
interesting, and potentially of clinical significance, that orally administered $P$ quinquefolius can also reverse postinfarction remodelling and heart failure when administered four weeks following infarction, when the hypertrophy, remodelling and left ventricular function have already been well-established (Moey et al. 2012). To reverse this process, thus producing reverse remodelling, is a major therapeutic challenge and difficult to achieve with most pharmacological agents yet it is an important attribute for the development of new treatments for heart failure (Hellawell and Margulies 2012; Rodrigues et al. 2016). It is possible that ginseng in general and $P$ quinquefolius in particular could be a useful adjunctive therapy for treating heart failure by initiating reverse remodelling in patients with established heart failure, particularly in combination with other therapies.

Although the coronary artery ligation model is principally related to left ventricular hypertrophy and left ventricular dysfunction, the effect of ginseng-related products was also determined in a number of studies using a model of right ventricular hypertrophy in the rat produced by administering monocrotaline, a plant-derived pyrrolizidine alkaloid which produces pulmonary artery remodelling and pulmonary hypertension resulting in right ventricular hypertrophy (Gomez-Arroyo et al. 2012). Ginsenoside Rblmarkedly inhibited and reversed right ventricular hypertrophy in rats treated with monocrotaline (Jiang et al. 2007a). Although mechanisms were not explored in detail in that study, the authors provided evidence for a reduction in calcineurin expression in ventricles from $\mathrm{Rb} 1$ treated animals, although activity of this peptidase, likely a more important parameter than expression levels, was not determined. When a preparation of total ginsenosides from $P$ ginseng, containing Rb1, Rg1, Re, Rd plus others, was administered to monocrotaline-treated rats a similar beneficial effect was observed although this effect was associated with a multiplicity of cell signalling effects including inhibition of calcineurin 
expression as was shown for Rb1 (Jiang et al. 2007a) as well as inhibition of activities of MAPK ERK and mitogen-activated protein kinase phosphatase-1 (MKP-1), the latter known as dual specificity phosphatase-1 that plays an important role in the deactivation of MAPKs (Qin et al. 2008). Interestingly, a NO synthase inhibitor had no effect on the antihypertrophic effect of total ginsenosides in this experimental model of right ventricular hypertrophy (Qin et al. 2008).

Other models of disease as well as a genetic model of heart failure have also been studied, as mentioned, to demonstrate efficacy of ginseng. For example, cardiomyopathy secondary to induction of type 1 diabetes in rats is a well-established model for diabetic cardiomyopathy resulting in heart failure. Ginseng administration to these animals improved cardiac function in the absence of any changes in blood glucose levels (Tsai et al. 2014). This suggests a direct effect of ginseng on myocardial remodelling which the authors attributed to increased expression of the transcriptional factor peroxisome proliferator-activated receptor alpha (PPAR $\alpha)$. The species of ginseng used was however not apparent in this report. However, the salutary effect of P quinquefolius was demonstrated in another study showing that its administration to mice with both type 1 and type 2 diabetes markedly improved metabolic status, reduced oxidative stress and reduced myocardial hypertrophy while improving cardiac function (Sen et al. 2013). When taken together, the results are encouraging as they suggest that ginseng could be an effective treatment for cardiac complications associated with diabetes including the attenuation of diabetic cardiomyopathy.

Two separate studies utilized the transgenic $\mathrm{cTnT}^{\mathrm{R} 141 \mathrm{~W}}$ mouse model of dilated cardiomyopathy to assess the potential benefit of ginsenoside Rb1. Treatment of these mice for four months with $\mathrm{Rb} 1$ resulted in substantially reduced myocardial remodelling, improved function and reduced mortality which were associated with reduced STAT3 activation (Zhao et al. 2010). The benefit 
associated with administering ginsenoside Rb1 to these animals was found to occur in a synergistic manner when treatment was combined with tetramethylpyrazine phosphate, an inhibitor of calcium/calmodulin/calmodulin-dependent protein kinase activity (Lu et al. 2012).

Recent studies from the author's laboratory have shown effective ability of orally administered $P$ quinquefolius to limit numerous indices of myocardial remodelling in rats treated with fourteen day infusion of the $\beta$ adrenergic agonist isoproterenol, administered via osmotic minipump implantation. A primary observation was the reduction in the hypertrophic response as well as improved left ventricular function as determined by serial echocardiography (Tang et al. 2016). These effects were also associated with diminished activation of the $\beta_{1}$ adrenergic signalling pathway in hearts of isoproterenol-treated animals. Thus $P$ quinquefolius appears to be an effective anti-adrenergic agent potentially of benefit for the treatment of conditions associated with excessive adrenergic stimulation.

Ginseng-containing multicomponent traditional Chinese herbal medications have also been found to reduce both experimental and clinical heart failure. Among these is Shengmai, a herbal medication consisting of $P$ ginseng, Ophiopogon japonicus and Schisandra chinensis. The clinical aspects of Shengmai are discussed below although this agent has been shown to reduce cardiomyopathy produced by adriamyacin administration to rats (You et al. 2006). Moreover, treatment of aged rats for six weeks with Shengmai improved their cardiac function through a mechanism involving alterations in calcium homeostasis involving enhanced calcium release from the sarcoplasmic reticulum (Zhang et al. 2008).

\section{Studies on hypertrophy using cultured cardiomyocytes}


Cultured ventricular myocytes are a useful model for examining the hypertrophic program and its underlying mechanisms which can contribute to the myocardial remodelling process that can result in the development of heart failure in the intact heart. A number of studies have reported that ginseng and various ginsenosides can reduce hypertrophy in response to a variety of hypertrophic factors such as endothelin-1 whose hypertrophic effects are reduced by trilinolein, a bioactive factor isolated from $P$ ginseng (Chen et al. 2005). This effect has been attributed to a reduction in ET-1-induced generation of reactive oxygen species (ROS) in cardiomyocytes (Chen et al. 2005). It has been suggested that as a consequence of ROS inhibition the resultant effect would be to attenuate both MAPK activation as well as the elevation in the protooncogene $c$-fos (Yang et al. 2005). Trilinolein has been shown to inhibit the hypertrophic effects of other agonists as well including angiotensin II and norepinephrine both purported to occur by inhibiting the production of ROS (Liu et al. 2004a, 2004b).

While inhibition of ROS generation is likely of importance in attenuating the hypertrophic effects of various agonists on cardiac myocytes by ginseng preparations, other mechanisms are also likely involved which may reflect both the nature of the agonist as well as the ginseng product used. For example, ginsenoside Rb1 has been shown to inhibit the hypertrophic effect of prostaglandin F2 $\alpha$ through a mechanism involving downregulation of the calcineurin-dependent pathway (Jiang et al. 2007b). However, a limitation of this study is that expression levels of calcineurin, NFAT and other transcriptional factors, and not activity levels were determined (Jiang et al. 2007b) with the latter likely reflecting a more appropriate index of the degree of calcineurin contribution to the hypertrophic process.

Another pathway which could represent a potential target for ginseng-induced inhibition of the hypertrophic response is the Ras homolog gene family, member A/Rho-associated, coiled-coil 
containing protein kinase (RhoA/ROCK) pathway which plays a major role in the regulation of cell function, particularly cytoskeleton dynamics as well as in various cardiac pathologies (Hartmann et al. 2015). Specifically, P quinquefolius was found to inhibit cardiomyocyte hypertrophy produced by the satiety-inducing adipokine leptin through a mechanism associated with RhoA/ROCK inhibition (Moey et al. 2011). This was found to occur via the inhibition of p115RhoGEF, a guanine nucleotide exchange factor which catalyzes the exchange of GDP to GTP resulting in RhoA activation (Moey et al. 2011).

\section{Clinical evidence for benefit by ginseng-related products}

In terms of demonstration of clinical efficacy, the first report, published in the Chinese literature, demonstrated that a combination of red ginseng with the cardiac glycoside digoxin produced the best benefit compared to either drug alone when administered to a small group of patients with severe heart failure, thus suggesting a potential additive effect of ginseng when used as adjunctive therapy (Ding et al. 1995). Since that initial report no studies have documented strong clinical evidence for therapeutic usefulness of ginseng for treating chronic heart failure based on randomized placebo-controlled large scale clinical trials. Nonetheless, there is other evidence using ginseng-containing preparations for potential clinical benefit based on studies carried out in China. Among these is the use of Shenmai, widely used Chinese medicine indicated for a large number of conditions and which appears to be devoid of major side effects (Zhang et al. 2010). Shenmai is an extract from red ginseng as well as the ophiopogon root from evergreen perennial plants grown throughout much of Asia. It is a widely used therapeutic agent in China and one which has been reported to improve left ventricular functions in patients with heart failure due to dilated cardiomyopathy (Zhang et al. 2002). Moreover, Shenmai improved diastolic function as assessed by Doppler imaging when administered to heart failure patients 
(Ma et al. 2010). The beneficial effects of Shenmai in heart failure patients has been proposed to be due to downregulation in the serum levels of the death receptors DR4 and DR5, which mediate the effect of tumour necrosis factor-related apoptosis-inducing ligand (TRAIL), a member of the tumour necrosis factor family of ligands thereby reducing the potential for apoptosis in patients with heart failure (Hong et al. 2005). Because of the complex nature of ginseng multicomponent preparations, it is likely that any benefit provided by Shenmai in heart failure patients involves other mechanisms.

As already alluded to one of the major problems in assessing the potential efficacy of Shenmai, and indeed other ginseng preparations, in the treatment of heart is related to the paucity of large scale randomized controlled clinical trials. Two meta-analyses of published small scale clinical trials provided cautionary evidence that Shenmai addition to existing heart failure medications provided some benefit in improving New York Heart Association (NYHA) functional classification (Chen et al. 2012; Zhou et al. 2014). Accordingly, both reports proposed further evaluation to confirm efficacy with higher quality and longer follow-up trials. However, a recently reported clinical study using a larger population group consisting of an initial enrolment of 240 patients supported the concept of a beneficial effect of Shenmai in NYHA Class II to IV heart failure patients. In that study, the primary benefit seen with Shenmai administration for one week in combination with standard therapy was an improvement in the NYHA classification score although left ventricular ejection fraction was actually not significantly improved (Xian et al. 2016). However, this study as well has limitations in view of the short treatment period and lack of long-term follow up in these patients.

Administration of Shenfu, a water extract of $P$ ginseng, to NYHA Class II to IV heart failure patients for fourteen days resulted in the improvement in a number of parameters (Wei et al. 
2015). These included improved quality of life scores as determined by a questionnaire as well as improved left ventricular function, reduced plasma cytokine levels and reduced indices of hepatic injury (Wei et al. 2015). As with other clinical studies using ginseng products a major limitation which confounds interpretation of the results is the small population (18 patients in treatment group) size and short treatment period employed in this trial.

\section{Conclusion and future directions}

The experimental evidence for the beneficial of ginseng and ginseng-related products for the treatment of heart failure and as an anti-hypertrophic factor is quite convincing based on a substantial amount of animal-derived data (summarized in Table 2). However, the clinical evidence for the use of ginseng products for the treatment of heart failure is generally based on rather small clinical trials with no evidence for large scale well-designed, randomized and placebo-controlled Phase 3 clinical trials. Undoubtedly, establishing such trials is challenging on many fronts, however these need to be performed in order to bring ginseng into the widespread clinical arena for treatment of heart failure. Also challenging, but perhaps of lesser importance is identification of the mechanisms underlying the salutary effects of ginseng on reducing the myocardial remodelling process. However, based on extensive evidence as discussed here, this is unlikely to reflect a single mechanism but instead involves modulation of numerous cellular processes. Two such key mechanisms, among others, is attenuation of $\beta_{1}$ adrenergic responses as well as inhibition of $\mathrm{Ca}^{2+}$-calmodulin dependent calcineurin activation likely secondary to NHE inhibition (Figure 1). Another important issue to consider when administering ginseng for the treatment of heart failure, and indeed for other cardiovascular conditions is the potential for drug interactions as many heart failure patients are on multiple pharmacotherapies. These potential drug interactions have not been extensively studied however with respect to anticoagulant 
administration to stroke patients it appears that ginseng has no effect on the anticoagulant effect of warfarin (Lee et al. 2008), although a cautionary note has been raised by one group of authors (Shao and Jia 2013). Clearly, substantial research is required in this area in order to fulfil the promise of ginseng as an effective adjunctive therapy for the treatment of heart failure.

\section{Acknowledgements}

Work from the authors' laboratory was supported by the Canadian Institutes of Health Research and the Ontario Ginseng Innovation and Research Consortium. The authors wish to acknowledge the contribution of colleagues and laboratory members who have contributed to these studies including Edmund Lui, Melissa Moey, Juan Guo, Xilan Tang, Eduardo MartínezAbundis, Cathy Huang, Venkatesh Rajapurohitam, Asad Zeidan, James Haist and Jenny Xue. 


\section{References}

Akao, T., Kida, H., Kanaoka, M., Hattori, M., and Kobashi, K. 1998. Intestinal bacterial hydrolysis is required for the appearance of compound $\mathrm{K}$ in rat plasma after oral administration of ginsenoside Rb1 from Panax ginseng. J. Pharm. Pharmacol. 50:1155-1160

Ambrosy, A.P., Fonarow, G.C., Butler, J., Chioncel, O., Greene, S.J., Vaduganathan, M., Nodari, S., Lam, C.S., Sato, N., Shah, A.N., and Gheorghiade M. 2014.The global health and economic burden of hospitalizations for heart failure: lessons learned from hospitalized heart failure registries. J. Am. Coll. Cardiol. 63: 1123-1133. doi: 10.1016/j.jacc.2013.11.053

Attele, A.S., Wu, J.A., and Yuan, C.S. 1999. Ginseng pharmacology: multiple constituents and multiple actions. Biochem. Pharmacol. 58: 1685-1693.

Breckenridge, R. 2010. Heart failure and mouse models. Dis. Model. Mech. 3: 138-143. doi: $10.1242 / \mathrm{dmm} .005017$

Chen, S.C., Cheng, J.J., Hsieh, M.H., Chu, Y.L., Kao, P.F., Cheng, T.H., and Chan, P. 2005. Molecular mechanism of the inhibitory effect of trilinolein on endothelin-1-induced hypertrophy of cultured neonatal rat cardiomyocytes. Planta Med. 71: 525-529.

Chen, J., Yao, Y., Chen, H., Kwong, J.S., and Chen, J. 2012. Shengmai (a traditional Chinese herbal medicine) for heart failure. Cochrane Database Syst. Rev. 11:CD005052. doi: 10.1002/14651858.CD005052.pub4

Deng, J., Lv, X.T., Wu, Q., and Huang, X.N. 2009. Ginsenoside Rg1 inhibits rat left ventricular hypertrophy induced by abdominal aorta coarctation: involvement of calcineurin and mitogen- 
activated protein kinase signalings. Eur. J. Pharmacol. 608: 42-47. doi:

10.1016/j.ejphar.2009.01.048

Deng, J., Wang, Y.W., Chen, W.M., Wu, Q., and Huang, X.N. 2010. Role of nitric oxide in ginsenoside $\operatorname{Rg}(1)$-induced protection against left ventricular hypertrophy produced by abdominal aorta coarctation in rats. Biol. Pharm. Bull. 33: 631-635.

Ding, D.Z., Shen, T.K., and Cui, Y.Z. 1995. Effects of red ginseng on the congestive heart failure and its mechanism. Zhongguo Zhong Xi Yi Jie He Za Zhi, 15: 325-327. (Chinese)

Frantz, S., Bauersachs, J., and Ertl, G. 2009. Post-infarct remodelling: contribution of wound healing and inflammation. Cardiovasc. Res. 81:474-481. doi: 110. Frey, N., Katus, H.A., Olson, E.N., and Hill, J.A. 2004. Hypertrophy of the heart: a new therapeutic target? Circulation, 109:1580-1589. 0.1093/cvr/cvn292

Garriques, S.S. 1854. On panaquilon, a new vegetable substance. Ann. Chem. Pharm. 90:231234. doi: $10.1002 /$ jlac. 18540900216

Gomez-Arroyo, J.G., Farkas, L., Alhussaini, A.A, Farkas, D., Kraskauskas, D., Voelkel, N.F., and Bogaard, H.J. 2012. The monocrotaline model of pulmonary hypertension in perspective. Am. J. Physiol. Lung Cell. Mol. Physiol. 302: L363-L369. doi: 10.1152/ajplung.00212.2011

Guo, J., Gan, X.T., Haist, J.V., Rajapurohitam, V., Zeidan, A., Faruq, N.S., Karmazyn, M. 2011. Ginseng inhibits cardiomyocyte hypertrophy and heart failure via NHE-1 inhibition and attenuation of calcineurin activation. Circ. Heart Fail. 4:79-88. doi:

10.1161/CIRCHEARTFAILURE.110.957969. 
Hartmann, S., Ridley, A.J., and Lutz, S. 2015. The Function of Rho-Associated Kinases ROCK1 and ROCK2 in the pathogenesis of cardiovascular disease. Front. Pharmacol. 6:276. doi: 10.3389/fphar.2015.00276

Heineke, J., and Molkentin, J.D. 2006. Regulation of cardiac hypertrophy by intracellular signalling pathways. Nat. Rev. Mol. Cell. Biol. 7: 589-600.

Hellawell, J.L., and Margulies, K.B. 2012. Myocardial reverse remodeling. Cardiovasc. Ther. 30: 172-181. doi: 10.1111/j.1755-5922.2010.00247.x

Hong, Y., Xie, W., and Chen, C.S. 2005. Effect of shengmai injection on TRAIL death receptor of patients with congestive heart failure. Zhongguo Zhong Xi Yi Jie He Za Zhi, 25: 1092-1095. (Chinese)

Houser, S.R., Margulies, K.B., Murphy, A.M., Spinale, F.G., Francis, G.S., Prabhu, S.D., Rockman, H.A., Kass, D.A., Molkentin, J.D., Sussman, M.A., and Koch, W.J. American Heart Association Council on Basic Cardiovascular Sciences, Council on Clinical Cardiology, and Council on Functional Genomics and Translational Biology. 2012. Animal models of heart failure: a scientific statement from the American Heart Association. Circ. Res. 111: 131-50. doi: 10.1161/RES.0b013e3182582523. Erratum in: Circ Res (2012) 111: e54.

Javadov, S., and Karmazyn M. 2007. Mitochondrial permeability transition pore opening as an endpoint to initiate cell death and as a putative target for cardioprotection. Cell. Physiol. Biochem. 20:1-22. 
Jiang, Q.S., Huang, X.N., Dai, Z.K., Yang, G.Z., Zhou, Q.X., Shi, J.S., and Wu, Q. $2007 a$. Inhibitory effect of ginsenoside $\mathrm{Rb} 1$ on cardiac hypertrophy induced by monocrotaline in rat. J. Ethnopharmacol. 111: 567-572.

Jiang, Q.S., Huang, X.N., Yang, G.Z., Jiang, X.Y., and Zhou, Q.X. 2007b. Inhibitory effect of ginsenoside $\mathrm{Rb}$ l on calcineurin signal pathway in cardiomyocyte hypertrophy induced by prostaglandin F2alpha. Acta Pharmacol. Sin. 28: 1149-1154.

Karikura, M., Miyase, T., Tanizawa, H., Takino, Y., Taniyama, T., and Hayashi, T. 1990. Studies on absorption, distribution, excretion and metabolism of ginseng saponins. V. The decomposition products of ginsenoside Rb2 in the large intestine of rats. Chem. Pharm. Bull. (Tokyo) 38: 2859-2861.

Karikura, M., Miyase, T., Tanizawa, H., Taniyama, T., and Takino, Y. 1991. Studies on absorption, distribution, excretion and metabolism of ginseng saponins. VII. Comparison of the decomposition modes of ginsenoside-Rb1 and $-\mathrm{Rb} 2$ in the digestive tract of rats. Chem. Pharm. Bull. 39: 2357-2361.

Karmazyn, M., Kilić, A., Javadov, S. 2008. The role of NHE-1 in myocardial hypertrophy and remodelling. J. Mol. Cell. Cardiol. 44: 647-653. doi: 10.1016/j.yjmcc.2008.01.005

Katz, A.M. 2000. The hypertrophic response: Programmed cell death. In Heart Failure. Pathophysiology, Molecular Biology, and Clinical Management. By A.M. Katz. Lippincott Williams \& Wilkins, Philadelphia, PA. pp 173-226. 
Kim, D.H. 2002. Herbal medicines are activated by intestinal microflora. Nat. Prod. Sci. 8:3543.

Kobashi, K., Akao, T. 1997. Relation of intestinal bacteria to pharmacological effects of glycosides. Bioscience Microflora, 16:1-7.

Lee, S.H., Ahn, Y,M., Ahn, S.Y., Doo, H.K., and Lee, B.C. 2008. Interaction between warfarin and Panax ginseng in ischemic stroke patients. J. Altern. Complement. Med. 14: 715-721. doi: 10.1089/acm.2007.0799

Liu, J.C., Cheng, T.H., Lee, H.M., Lee, W.S., Shih, N.L., Chen, Y.L., Chen. J.J., and Chan, P. 2004a. Inhibitory effect of trilinolein on angiotensin II-induced cardiomyocyte hypertrophy. Eur. J. Pharmacol. 484: 1-8.

Liu, J.C., Chan, P., Chen, J.J., Lee, H.M., Lee, W.S., Shih, N.L., Chen, Y.L., Hong, H.J., and Cheng T.H. 2004b. The inhibitory effect of trilinolein on norepinephrine-induced beta-myosin heavy chain promoter activity, reactive oxygen species generation, and extracellular signalregulated kinase phosphorylation in neonatal rat cardiomyocytes. J. Biomed. Sci. 11: 11-18. Lu, D., Shao, H.T., Ge, W.P., Liu, N., Zhang, X., Ma, C,M., Qin, C., and Zhang, L.F. 2012. Ginsenoside-Rb1 and tetramethylpyrazine phosphate act synergistically to prevent dilated cardiomyopathy in cTnTR141W transgenic mice. J. Cardiovasc. Pharmacol. 59: 426-433. doi: 10.1097/FJC.0b013e318249509e

Ma, R.G., Wang, C.X., Shen, Y.H., Wang, Z.Q., Ma, J.H., Huang, L.S. 2010. Effect of Shenmai Injection on ventricular diastolic function in patients with chronic heart failure: An assessment by tissue Doppler imaging. Chin. J. Integr. Med. 16: 173-175. doi: 10.1007/s11655-010-0173-1 
Moey, M., Rajapurohitam, V., Zeidan, A., and Karmazyn, M. 2011. Ginseng (Panax quinquefolius) attenuates leptin-induced cardiac hypertrophy through inhibition of p115Rho guanine nucleotide exchange factor-RhoA/Rho-associated, coiled-coil containing protein kinasedependent mitogen-activated protein kinase pathway activation. J. Pharmacol. Exp. Ther. 339: 746-756. doi: 10.1124/jpet.111.182600

Moey, M., Gan, X.T., Huang, C.X., Rajapurohitam, V., Martínez-Abundis, E., Lui, E.M., and Karmazyn, M. 2012. Ginseng reverses established cardiomyocyte hypertrophy and postmyocardial infarction-induced hypertrophy and heart failure. Circ. Heart Fail. 5: 504-514. doi: 10.1161/CIRCHEARTFAILURE.112.967489

Morishita, S., Shoji, M., Oguni, Y., Ito, C., Noguchi, K., and Sakanashi, M. 1992a. Effects of "kyushin", a drug containing toad venom, on experimental congestive heart failure in rabbits. Am. J. Chin. Med. 20: 83-89.

Morishita, S., Shoji, M., Oguni, Y., Ito, C., Higuchi, M., and Sakanashi, M. $1992 b$. Pharmacological actions of "kyushin," a drug containing toad venom: cardiotonic and arrhythmogenic effects, and excitatory effect on respiration. Am. J. Chin. Med. 20: 245-256.

Muders, F., and Elsner, D. 2000. Animal models of chronic heart failure. Pharmacol. Res. 41: 605-612.

Odani, T., Tanizawa, H., and Takino, Y. 1983a. Studies on the absorption, distribution, excretion and metabolism of ginseng saponins. II. The absorption,distribution and excretion of ginsenoside Rg1 in the rat. Chem. Pharm. Bull. (Tokyo) 31:292-298. 
Odani, T., Tanizawa, H., Takino, Y. 1983b. Studies on the absorption, distribution, excretion and metabolism of ginseng saponins. III. The absorption, distribution and excretion of ginsenoside Rb1 in the rat. Chem. Pharm. Bull. (Tokyo) 31:1059-1066.

Opie, L.H., Commerford ,P.J., Gersh, B.J., and Pfeffer, M.A. 2006. Controversies in ventricular remodelling. Lancet, 367:356-367.

Panther, F., Williams, T., and Ritter, O. 2009. Inhibition of the calcineurin-NFAT signalling cascade in the treatment of heart failure. Recent Pat. Cardiovasc. Drug Discov. 4:180-186.

Patten, R.D., and Hall-Porter, M.R. 2009. Development of therapeutics for heart failure. Small animal models of heart failure: Development of novel therapies, past and present. Circ. Heart Fail. 2:138-144. doi:10.1161/CIRCHEARTFAILURE.108.839761

Qin, N., Gong, Q.H., Wei, L.W., Wu,Q., and Huang, X.N. 2008. Total ginsenosides inhibit the right ventricular hypertrophy induced by monocrotaline in rats. Biol. Pharm. Bull. 31: 15301535.

Rodrigues, P.G., Leite-Moreira, A.F., and Falcão-Pires, I. 2016. Myocardial reverse remodeling: how far can we rewind? Am. J. Physiol. Heart Circ. Physiol. 310: H1402-H1422. doi: 10.1152/ajpheart.00696.2015

Sen, S., Chen, S., Wu, Y., Feng, B., Lui E.K., and Chakrabarti, S. 2013. Preventive effects of North American ginseng (Panax quinquefolius) on diabetic retinopathy and cardiomyopathy. Phytother. Res. 27: 290-298. doi: 10.1002/ptr.4719 
Shao, J. and Jia, L. 2013. Potential serious interactions between nutraceutical ginseng and warfarin in patients with ischemic stroke. Trends Pharmacol. Sci. 34: 85-86. doi:

10.1016/j.tips.2012.11.008

Shibata, S., Tanaka, O., NagaI, M., and Ishii, T. 1963a. Studies on the constituents of Japanese and Chinese crude drugs. XII. Panaxadiol, a sapogenin of ginseng roots. Chem. Pharm. Bull. (Tokyo) 11:762-765. doi: 10.1248/cpb.11.762

Shibata, S., Fujita, M., Itokawa, H., Tanko, O, and Ishii, T. 1963b. Studies on the constituents of Japanese and Chinese Crude Drugs. XI. Panaxadiol, a sapogenin of ginseng roots (1). Chem. Pharm. Bull. (Tokyo) 11:759-761.

Shibata, S., Ando, T., Tanaka, O., Meguro, Y., Sôma, K., and Iida, Y. 1965a. Saponins and sapogenins of Panax ginseng C.A. Meyer and some other Panax spp. Yakugaku Zasshi, 85:753755.

Shibata, S., Tanaka, O., Soma, K., Aando, T., Iida, Y., and Nakamura, H. 1965b. Studies on saponins and sapogenins of ginseng. The structure of panaxatriol. Tetrahedron Lett. 42:207-213.

Shimizu, I., and Minamino, T. 2016. Physiological and pathological cardiac hypertrophy. J. Mol. Cell. Cardiol. 97:245-262. doi: 10.1016/j.yjmcc.2016.06.001

Shin, B.K., Kwon, S.W., and Park, J.H. 2015. Chemical diversity of ginseng saponins from Panax ginseng. J. Ginseng Res. 39: 287-298. doi: 10.1016/j.jgr.2014.12.005.

Sun, Y. 2009. Myocardial repair/remodelling following infarction: roles of local factors. Cardiovasc. Res. 81:482-490. doi: 10.1093/cvr/cvn333 
Tang, X., Gan, X.T., Rajapurohitam, V., Huang, C.X., Xue, J., Lui, E.K., and Karmazyn, M. 2016. North American ginseng (P. quinquefolius) suppresses $\beta$ adrenergic-dependent signalling, hypertrophy and cardiac dysfunction. Can. J. Physiol. Pharmacol. 94:1325-1335.

Tsai, C.C., Chan, P., Chen, L.J., Chang, C.K., Liu, Z., and Lin, J.W. 2014. Merit of ginseng in the treatment of heart failure in type 1-like diabetic rats. Biomed. Res. Int. 2014:484161. doi: $10.1155 / 2014 / 484161$

Tu, J.V., Nardi, L., Fang, J., Liu, J., Khalid, L., and Johansen, H.; Canadian Cardiovascular Outcomes Research Team. 2009. National trends in rates of death and hospital admissions related to acute myocardial infarction, heart failure and stroke, 1994-2004. Can. Med. Assoc. J. 180:E118-E125. doi: 10.1503/cmaj.081197

Wei, H., Wu, H., Yu, W., Yan, X., and Zhang, X. 2015. Shenfu decoction as adjuvant therapy for improving quality of life and hepatic dysfunction in patients with symptomatic chronic heart failure. J. Ethnopharmacol. 169:347-355. doi: 10.1016/j.jep.2015.04.016

Xian, S., Yang, Z., Lee, J., Jiang, Z., Ye, X., Luo, L., Jin, L., Yang, T., Ye, S., and Lu, D. 2016. A randomized, double-blind, multicenter, placebo-controlled clinical study on the efficacy and safety of Shenmai injection in patients with chronic heart failure. J. Ethnopharmacol. 186:136142. doi: 10.1016/j.jep.2016.03.066

Yang, H.Y., Liu, J.C., Chen, Y.L., Chen, C.H., Lin, H., Lin, J.W., Chiu, W.T., Chen, J.J., and Cheng, T.H. 2005. Inhibitory effect of trilinolein on endothelin-1-induced c-fos gene expression in cultured neonatal rat cardiomyocytes. Naunyn Schmiedebergs Arch. Pharmacol. 372: 160-167. 
You, J.S., Huang, H.F., and Chang, Y. L. 2005. Panax ginseng reduces adriamycin-induced heart failure in rats. Phytother. Res. 19: 1018-1022.

You, J.S., Huang, H.F., Chang, Y.L., Lee, Y.S. 2006. Sheng-mai-san reduces adriamycininduced cardiomyopathy in rats. Am. J. Chin. Med. 34: 295-305.

Zhang, Y.C., Chen, R.M., and Zhao, M.H. 2002. Effect of shengmai injection on hemodynamics in patients with dilated cardiomyopathy. Zhongguo Zhong Xi Yi Jie He Za Zhi, 22: 277-179. (Chinese)

Zhang, G.Q., Wang, H., Liu, W.T., Dong, H., Fong, W.F., Tang, L.M., Xiong, Y.H., Yu, Z.L., and Ko, K.M. 2008. Long-term treatment with a Chinese herbal formula, Sheng-Mai-San, improves cardiac contractile function in aged rats: the role of $\mathrm{Ca} 2+$ homeostasis. Rejuvenation Res. 11: 991-1000. doi: 10.1089/rej.2008.0771

Zhang, L., Hu, J., Xiao, L., Zhang, Y., Zhao, W., Zheng, W., and Shang, H. 2010. Adverse drug reactions of Shenmai injection: a systematic review. J. Evid. Based Med. 3: 177-182. doi: 10.1111/j.1756-5391.2010.01089.x

Zhang, Y.J., Zhang, X.L., Li, M.H., Iqbal, J., Bourantas, C.V., Li, J.J., Su, X.Y., Muramatsu, T., Tian, N.L., Chen, S.L. 2013. The ginsenoside Rg1 prevents transverse aortic constrictioninduced left ventricular hypertrophy and cardiac dysfunction by inhibiting fibrosis and enhancing angiogenesis. J. Cardiovasc. Pharmacol. 62: 50-57. doi: 10.1097/FJC.0b013e31828f8d45

Zhao, H., Lv, D., Zhang, W., Dong, W., Feng, J., Xiang, Z., Huang, L., Qin, C., and Zhang, L. 2010. Ginsenoside-Rb1 attenuates dilated cardiomyopathy in cTnT(R141W) transgenic mouse. J. Pharmacol. Sci. 112: 214-222. 
Zhou, Q., Qin, W,Z., Liu, S.B., Kwong, J.S., Zhou, J., and Chen, J. 2014. Shengmai (a traditional Chinese herbal medicine) for heart failure. Cochrane Database Syst. Rev. 14: CD005052. doi: 10.1002/14651858.CD005052.pub5 
Figure 1. Two primary pathways of cardiomyocyte hypertrophy shown to be inhibited by ginseng and/or ginsenosides. The left part of the figure illustrates $\beta_{1}$ adrenergic responses which results in protein kinase A (PKA) activation through initial activation of the Gs protein-coupled receptor. This in turn activates the transcriptional factor cAMP response element-binding protein (CREB) which initiates transcription resulting in the hypertrophic response. This pathway has been shown to be inhibited by $P$ quinquefolius although the precise site of action of this ginseng species has not been elucidated (Tang et al. 2016). The right side of this figure depicts calcineurin-dependent hypertrophic responses in response to activation of Gq-coupled receptors such as those for angiotensin II, endothelin-1 and $\alpha_{1}$-adrenergic agonists including norepinephrine or phenylephrine. Activation of these receptors results in activation of the sodium-hydrogen exchanger (NHE) which then results in a sodium-calcium exchange (NCX) dependent elevation in intracellular $\mathrm{Ca}^{2+}$ concentrations with the resulting $\mathrm{Ca}^{2+}$-calmodulin complex activating calcineurin. Calcineurin-dependent dephosphorylation of transcriptional factor nuclear factor of activated T cells (NFAT) allows the latter to be transported into nuclei thereby stimulating transcription and the hypertrophic process. A number of studies have shown that ginsenosides Rg1 (Deng et al. 2009) and Rb1 (Jiang et al. 2007a) as well as both $P$ ginseng (Qin et al. 2008) and P quinquefolius can target the calcineurin pathway potentially by inhibiting NHE expression and activity (Guo et al. 2011). See text for further discussion. Figure was created using the Motifolio Power Point Tool Kit. 


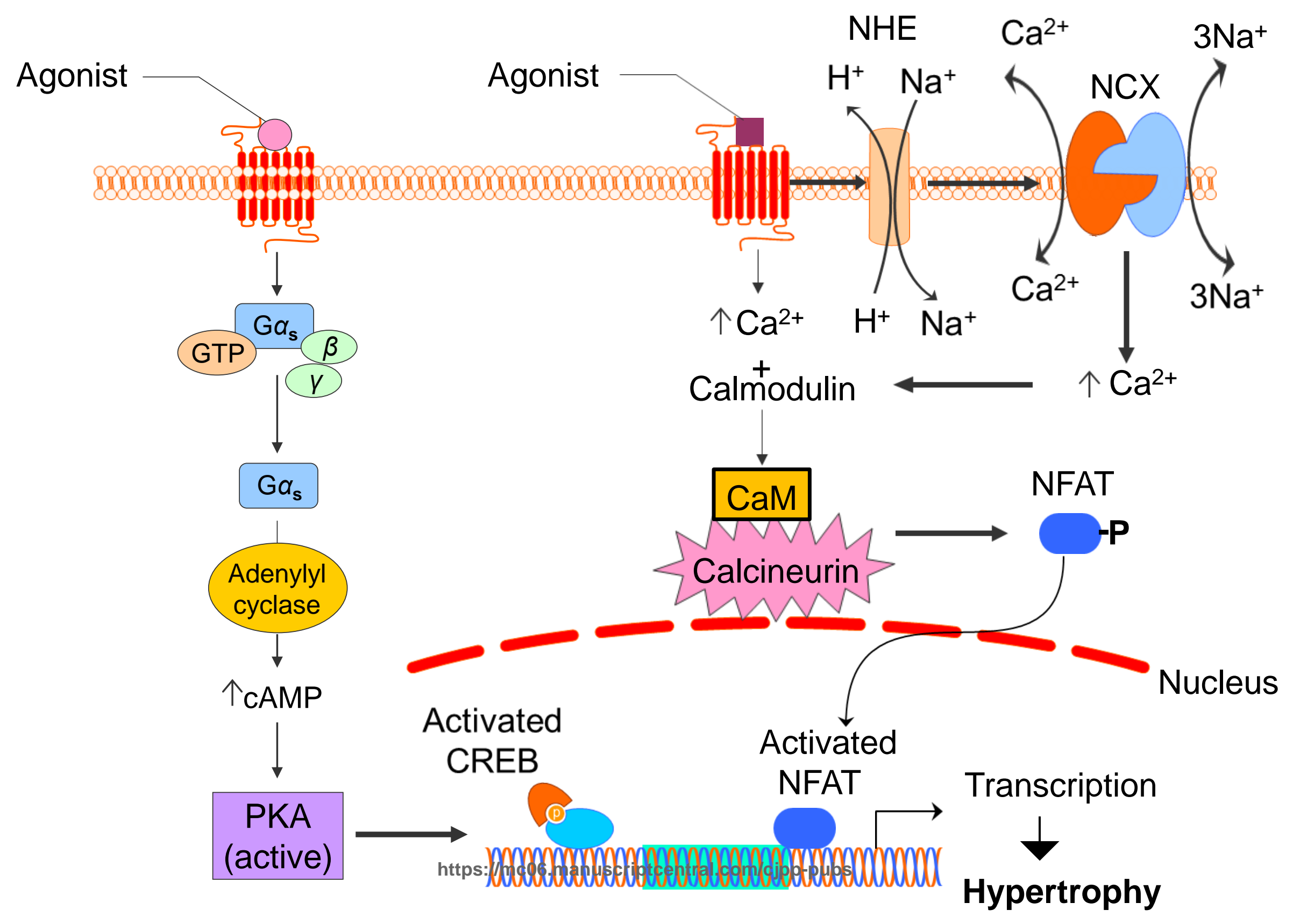


Table 1. Some ginseng species and their major saponins

\begin{tabular}{ll}
\hline Ginseng & Primary \\
\hline Panax ginseng & $\mathrm{Rg} 1, \mathrm{Re}, \mathrm{Rb} 1$ \\
Panax ginseng (Korean) & $\mathrm{Rb} 1, \mathrm{Rg} 1, \mathrm{Rb} 1, \mathrm{Rb} 2, \mathrm{Rf}$, \\
Panax quinquefolius & $\mathrm{Rb} 1, \mathrm{Rd}, \mathrm{Re}$, \\
Panax notoginseng & $\mathrm{Rb} 1, \mathrm{Rd}, \mathrm{Rg} 1, \mathrm{Ra} 3, \mathrm{Rf}$ \\
P vietnamensis & Majonoside R2 \\
\hline
\end{tabular}


Table 2. Experimental evidence for reduction in hypertrophy and heart failure by ginseng and ginseng-related products in various experimental models

\begin{tabular}{|c|c|c|c|c|}
\hline Experimental model & Treatment & Primary effects & Primary mechanism & Reference \\
\hline \multicolumn{5}{|l|}{ In vivo studies } \\
\hline $\begin{array}{l}\text { Rabbit protease } \\
\text { application to ventricle }\end{array}$ & Kyushin & 个LV function & Not studied & Morishita et al. $1992 a$ \\
\hline Adriamycin-treated rats & $\begin{array}{l}\text { P ginseng } \\
\text { Shengmai }\end{array}$ & $\begin{array}{l}\uparrow L V \text { function, } \downarrow \text { mortality } \\
\uparrow L V \text { function, } \downarrow \text { mortality }\end{array}$ & $\begin{array}{l}\text { Antioxidant } \\
\text { Antioxidant }\end{array}$ & $\begin{array}{l}\text { You et al. } 2005 \\
\text { You et al. } 2006\end{array}$ \\
\hline Rat TAC & Ginsenoside Rg1 & $\begin{array}{l}\downarrow \text { hypertrophy } \\
\uparrow L V \text { function, } \downarrow \text { fibrosis }\end{array}$ & $\begin{array}{l}\downarrow \text { MAPK, } \downarrow \text { calcineurin, } \uparrow \text { NO } \\
\uparrow \text { coronary angiogenesis }\end{array}$ & $\begin{array}{l}\text { Deng et al. 2009, } 2010 \\
\text { Zhang et al. } 2013\end{array}$ \\
\hline Rat CAL & P quinquefolius & $\begin{array}{l}\downarrow \text { hypertrophy, } \uparrow L V \text { function } \\
\text { Reversal of remodelling }\end{array}$ & $\begin{array}{l}\downarrow N H E-1, \downarrow \text { calcineurin } \\
\downarrow \text { calcineurin }\end{array}$ & $\begin{array}{l}\text { Guo et al. 2011) } \\
\text { Moey et al. } 2012\end{array}$ \\
\hline \multirow{2}{*}{$\begin{array}{l}\text { Rat monocrotaline- } \\
\text { induced RVH }\end{array}$} & Ginsenoside Rb1 & $\downarrow$ hypertrophy & $\downarrow$ calcineurin & Jiang et al. $2007 a$ \\
\hline & P ginseng & $\downarrow$ hypertrophy & $\downarrow$ calcineurin, $\downarrow$ ERK, $\downarrow$ MKP-1 & Qin et al. 2008 \\
\hline \multirow{2}{*}{$\begin{array}{l}\text { Rat diabetic cardiomyopathy } \\
\text { (species not indicated) }\end{array}$} & Ginseng & 个LV function & $\downarrow P P A R \alpha$ & Tsai et al. 2014 \\
\hline & $\begin{array}{l}P \text { quinquefolius } \\
\downarrow \text { hypertrophy }\end{array}$ & 个LV function, $\uparrow$ metabolism & $\downarrow$ oxidative stress & Sen et al. 2013 \\
\hline $\mathrm{cTn}^{\mathrm{R} 141 \mathrm{~W}}$ DCM Mice & Ginsenoside Rb1 & $\begin{array}{l}\uparrow L V \text { function, } \downarrow \text { mortality } \\
\downarrow \text { remodelling }\end{array}$ & $\downarrow$ STAT3 & $\begin{array}{l}\text { Zhao et al. 2010; } \\
\text { Lu et al., } 2012\end{array}$ \\
\hline
\end{tabular}


Rat isoproterenol infusion

P quinquefolius

Trilinolein

Trilinolein

Ginsenoside Rb1

P quinquefolius

ET-1 treated myocytes

Ang II-treated myocytes

NE-treated myocytes

PGF2 $\alpha$-treated myocytes

Leptin-treated myocytes $\uparrow L V$ function, $\downarrow$ mortality

$\downarrow$ hypertrophy

\section{In vitro studies}

$\downarrow$ hypertrophy

$\downarrow$ hypertrophy

$\downarrow$ hypertrophy

$\downarrow$ hypertrophy

$\downarrow$ hypertrophy $\downarrow$ adrenergic signaling

Tang et al. 2016

Ang II, angiotensin II; CAL, coronary artery ligation; cTnTR141W DCM Mice; transgenic mice with cardiac troponin T mutation demonstrating dilated cardiomyopathy; ERK, extracellular signal-regulated kinases; ET-1, endothelin 1; LV, left ventricle; MKP-1, mitogen-activated protein kinase phosphatase-1; NE, norepinephrine; NHE-1, sodium hydrogen exchanger isoform 1; p115RhoGEF, p115Rho guanine nucleotide exchange factor, PGF2 $\alpha$, prostaglandin F2 $\alpha$; ROS, reactive oxygen species; RVH, right ventricle hypertrophy; TAC, thoracic aorta coarctation. 